\title{
The impact of order and aspect in processing of causally linked sentences
}

\author{
Joanna Blochowiak $^{1}$, Jacques Moeschler ${ }^{1}$, Thomas Castelain ${ }^{2}$ \\ ${ }^{1}$ Department of Linguistics, University of Geneva, Switzerland \\ ${ }^{2} \mathrm{~L} 2 \mathrm{C} 2$, Institute of Cognitive Science, Lyon, France (now University of Costa Rica) \\ https://doi.org/10.36505/ExLing-2010/03/0004/000124
}

\begin{abstract}
This paper presents experimental studies that investigate two issues related to the expression of causality in French: (i) what is the impact of order (cause-consequence vs. consequence-cause) in the processing of causally linked sentences without connectives and (ii) how, if at all, the aspectual distinction influences the nature of causal relations and their processing. Our hypothesis is that the consequence-cause order is processed faster, as it is the order imposed by the paradigmatic causal connective parce que (because). The differences in reading time confirmed our hypothesis for weakly associated causes and consequences. The experiments also showed that the aspectual contrast between events and states affects the nature of causal relation itself and plays a considerable role in the processing of causality.
\end{abstract}

Key words: causality, order, aspect, event, state

\section{Causal discourse: order and aspect}

The starting point of our experimental investigation consists of a rather simple observation. Most languages describe causal relations with the help of a paradigmatic causal connective, like parce que (because) in French, in consequence-cause order only. For instance, when we hear the sentence "John fell because Mary pushed him" we understand that John's falling is the consequence of Mary's pushing him. Nevertheless, the cause-consequence order, which matches the occurrence of events in reality, is also available in natural language with or without connectives. The following sentence with the connective because "The neighbours are home because the lights are on" involves the causeconsequence order but, interestingly, does not have a canonical causal interpretation. It is a typical example of so called inferential (Moeschler 1989) or epistemic (Sweetser 1990) reading in which the speaker makes a causal guess on the basis of the available evidence. So, it appears that the consequence-cause order is predominant in association with the canonical causal uses of connectives like because. The question is whether it is just an arbitrary feature of the language or if there is a cognitive motivation behind it.

Also, in discourse semantics (Asher \& Lascarides 2003, farther SDRT), where the speaker must infer a relation to hold between sentences without

ExLing 2010: Proceedings of 3rd Tutorial and Research Workshop on Experimental Linguistics, 25-27 August, Athens, Greece 
connectives in order for a discourse to make sense, the two give rise to two distinct discourse relations. The cause-consequence order is identified with a cause seen as a result ('Result' in SDRT terms) whereas the consequence-cause order gives rise to the causal inference ('Explanation', in SDRT terms). Thus, the existence of two distinct discourse relations might indicate two different processes of understanding. One recent study (Rohde \& Horton, 2010) which showed that discourse relations are subject to the expectationdriven processing, suggests that this hypothesis is on the right track.

Contrary to some earlier studies on causal reasoning that provide evidence for the subjects' preference for the reasoning from cause to effect (consequence in our terms) (Ahn \& Nosek 1998, Waldmann 2000 and 2001), our hypothesis is that the consequence-cause order has some cognitive advantage and, thus, will be processed faster as opposed to the cause-consequence order.

The second point of our experimental study aims at verifying if different types of eventualities participating in causal relations (here we limit ourselves to the opposition events vs. states) manifest themselves in differences in their causal power that would be perceptible in causal processing. Theoretically, the differences between events and states can be quite well captured in terms of aspect (Vendler 1957). In their time dimension, events are approximately point-like entities which are dynamic and have a culmination point whereas states are non dynamic, homogenous (i.e. every part of a given state basically resembles to any other part) and extended in time. It seems that these aspectual differences also affect the nature of the causal relation itself. If I say that "I have a headache because the atmospheric pressure is low", we understand that I have a headache as long as the atmospheric pressure is low, which means that one state causally support the other while in a simple event-to-event causality one event works like a causal trigger for another event (Blochowiak 2010). The question related to this fact is whether this ontologically fundamental contrast has an impact on the nature of causal relations and whether these differences play a role in the processing of causality.

\section{Experimental design}

In order to test the two hypotheses we designed two experiments. The first one, which was conducted in collaboration with the L2C2 (ISC, Lyon), tested the event-condition for both orders: causeconsequence and consequence-cause. The second one, carried out at the University of Geneva, tested the state-condition for both orders. Three similar phases can be distinguished for both experiments.

In the first phase, for each experiment a list of about 40 sentences was created describing the situations involving events (e.g.: "Mary pushed John") for the event-condition and the situations involving states 
(e.g.: "John is sick") for the state-condition. The examples were chosen in such a way that the situation described by each sentence could be treated as a cause or as a consequence in a causal chain. For instance, "John is sick" can be a cause for "John doesn't go to school" or it can be a consequence for "John has eaten too much".

In the second phase, these lists of sentences were presented to participants as stimuli for answers. Each participant received a list of sentences with one of two following instructions: Please, provide a cause for the following sentences or Please, provide a consequence for the following sentences. In this way we obtained two sentence sequences corresponding to the causeconsequence order (e.g.: "Paul took his medicine. He is better now.") and consequence-cause order (e.g.: "Paul took his medicine. He was ill.").

In so constructed data set we observed that for some stimuli the same causes and consequences were frequently given by different participants and for some others not. To give a more fine-grained analysis of the resulting pattern we created the parameter association force which measures how similar the responses to one particular sentence are. The association force received the value strong if for the same sentence participants gave more than $50 \%$ of the same responses (we allowed certain variations in wording); otherwise its value was considered weak. For instance, if for the sentence "John is sick", more than $50 \%$ of participants gave the response "He has eaten too much" as cause then the force between "John is sick" and "He has eaten too much" was judged strong within the test 'cause'. Taking the association force parameter into account, we built up a list of sequences of two sentences weakly and strongly associated in both cause-consequence and consequence-cause orders for both event- and state-conditions.

In the third phase, the four resulting combinations of 2-sentence sequences (consequence-cause strong, consequence-cause weak, causeconsequence strong, cause-consequence weak) served as the input to reading time tests performed with E-Prime.

\section{Results and conclusions}

The ANOVA revealed a main effect of association force $(\mathrm{p}<.01)$ and that the factor order does intervene in a significant way with the association force $(\mathrm{p}<.05)$. For event-condition, there is no statistically significant difference between the reading times in two orders when the association force is strong whereas when the association force is weak the sentences are read faster in consequence-cause order.

A different picture arises from the preliminary results for the statecondition. In weak associations the sequences are read much more slowly in the consequence-cause order than in the cause-consequence order. Regarding the strong associations, there is no significant difference in reading time between the two orders. 
The results for the event-condition partially confirm our hypothesis of faster processing of the consequence-cause order (the consequence-cause order is read faster in weak associations). The results for the state-condition pinpoint the importance of aspectual differences. States which have fundamentally different aspectual properties from events also have a different impact on the cognitive processing of causality.

\section{Acknowledgements}

The authors would like to thank all the researchers from L2C2 (ISC, Lyon) who took part in the first experiment (event-condition): Coralie Chevalier, Jean-Baptiste Van der Henst and Isabelle Tapiero.

\section{References}

Ahn, W.K., Nosek B.A. 1998. Heuristics used in reasoning with multiple causes and effects. Proceedings of the 20th Annual Conference of the Cognitive Science Society, Mahwah, Erlbaum Associates, 24-29.

Asher, N., Lascarides A. 2003. Logic of Conversation. Cambridge. Cambridge Univesity Press.

Blochowiak, J. 2010. La relation causale et ses relata. NCLF 29, to appear.

Moeschler, J. 1989. Modélisation du dialogue: représentation de l'inférence argumentative, Paris, Hermès.

Moeschler, J. 2000. L'ordre temporel est-il naturel?. In Moeschler J. and Béguelin, M.J. (eds.), Référence temporelle et nominale, Berne, Peter Lang, 71-105.

Moeschler, J. 2003. L'expression de la causalité en français. CLF 25, 11-42.

Moeschler, J. et al. 2006. Le raisonnement causal: de la pragmatique du discours à la pragmatique expérimentale, NCLF 27, 241-262.

Sweetser, E. 1990. From Etymology to Pragmatics. Metaphorical and Cultural Aspects of Semantic Structure. Cambridge. Cambridge University Press.

Vendler, Z. 1957. Verbs and Times. Reprint in Z. Vendler, Linguistics in Philosophy, Ithaca: Cornell University Press, 1967, pp. 97-121.

Waldman, M.R. 2000. Competition among causes but not effects in predictive and diagnostic learning. Journal of Experimental Psychology: Learning, Memory and Cognition 26(1), 53-76.

Waldman, M.R. 2001. Predictive versus diagnostic causal learning: evidence from an overshadowing paradigm. Psychonomic Bulletin \& Review 8, 600-608. 